\title{
OPTICAL AND VLBI POLARIZATION MEASUREMENTS OF
}

\author{
AGN \\ D. C. GABUZDA \\ Department of Physics and Astronomy, University of Calgary \\ and \\ M. L. SITKO \\ Department of Astronomy, University of Cincinnati
}

\begin{abstract}
One of the most promising approaches to unravelling the relationship between the optical and radio emission in AGN is to obtain nearly simultaneous optical and VLBI polarization data. We have obtained such data for five AGN $(0735+178$, OJ 287, 1219+285, 3C 279, and BL Lac). These data suggest that there is a direct link between the optical and radio polarized emission, and that frequently the optical polarization of AGN is associated with the emergence of new VLBI components.
\end{abstract}

3C 279 is an OVV quasar, while the other four sources listed above are BL Lacertae objects. The continua of these sources and other "blazars" are dominated by nonthermal emission which is variable and highly polarized at optical-radio wavelengths. It is believed that this non-thermal emission is associated with the relativistic jets which are known to exist in these sources, but details of the jet structure and physics are still very uncertain. We use $\chi_{o p t}, \chi_{c}$, and $\chi_{j}$ to refer to the optical, VLBI core, and VLBI jet polarization position angles.

In every one of the five AGN for which we have data, there is evidence for a link between $\chi_{o p t}$ and either $\chi_{c}$ or $\chi_{j}$. In each of the BL Lacertae objects, the correspondance seems to be between $\chi_{\text {opt }}$ and $\chi_{c}$, while $\chi_{\text {opt }}$ in 3 C 279 (which was experiencing a large polarized outburst during our observations) is aligned with $\chi_{j}$ in a newly emerging jet component. These results suggest a much closer connection between the optical and radio emission than has usually been expected; the most likely origin for this connection is cospatiality of the optical and radio emission regions. Our data for OJ 287, for which we have two epochs a year apart, are particularly intriguing. Between the two epochs, $\chi_{j}$ was roughly constant while $\chi_{c}$ rotated by some $60^{\circ}$; this rotation in $\chi_{c}$ was found to be a precursor to the birth of a new VLBI component. Although there is no obvious relation between $\chi_{\text {opt }}$ and $\chi_{c}$ at either epoch, the difference between these two angles is the same at the two epochs; i.e., $\chi_{\text {opt }}$ appears to have experienced the same rotation as $\chi_{c}$. Thus, our results for both $\mathrm{OJ} 287$ and $3 \mathrm{C} 279$ both point towards an association between the optical polarization and the birth and emergence of new VLBI components. We suggest that a significant amount of the optical polarization in AGN is generated in such components, many of which are probably energetic, compact relativistic shocks.

A more thorough presentation and discussion of these data will be given in a paper by Sitko \& Gabuzda, to be submitted to the Astronomical Journal in October 1993.

T. J.-L. Courvoisier and A. Blecha: Multi-Wavelength Continuum Emission of AGN, 469.

(C) 1994 IAU. Printed in the Netherlands. 